\title{
A new method for measuring diffusion coefficient of gases in liquids by PLIF
}

\author{
JIMENEZ Mélanie ${ }^{\mathrm{a}^{*}, \mathrm{~b}, \mathrm{c}}$, DIETRICH Nicolas ${ }^{\mathrm{a}, \mathrm{b}, \mathrm{c}}$, HEBRARD Gilles $^{\mathrm{a}, \mathrm{b}, \mathrm{c}}$ \\ aniversité de Toulouse; INSA,UPS,INP; LISBP 135 Avenue de Rangueil, F-31077 Toulouse, France \\ bINRA, UMR792, Ingénierie des Systèmes Biologiques et des Procédés F-31400 Toulouse, France \\ ${ }^{\mathrm{c}} \mathrm{CNRS}, \mathrm{UMR} 5504$ F-31400 Toulouse, France
}

\begin{abstract}
Gas-liquid mass transfer is a major issue in engineering processes such as wastewater treatment or biogas production since this phenomenon is directly linked to their design and efficiency. In recent years, much research has been done in this area but some gaps still remain in our knowledge of gas-liquid transfer, in particular concerning molecular diffusivity. The determination of molecular diffusivity is commonly based on empirical correlations, such as the widely used Wilke and Chang (1955) expression, valid under specific conditions and with relatively high uncertainties. In the present work, an innovative and promising technique is proposed to determine diffusion coefficients of gases in liquids. This technique is based on visualizing and quantifying oxygen diffusion across a flat gas-liquid interface, in a Newtonian medium, using Planar Laser Induced Fluorescence (PLIF) with inhibition. Particle Image Velocimetry (PIV) experiments were conducted to confirm the hydrodynamic flow field in the liquid phase. Results included the visualization of oxygen diffusion over time and the quantification of this visualization. The oxygen diffusivity thus determined is in agreement with values found in the literature.
\end{abstract}

Key-words: Diffusion coefficient, gas/liquid interface, PLIF, PIV

\section{Introduction}

Since the twentieth century, various research works based on chemical methods (Jamnongwong 2010), microprobes (Chu 1992) or Planar Laser Induced Fluorescence (PLIF) have been conducted to characterize, visualize and quantify gas-liquid transport phenomena. A better understanding of these phenomena is of prime importance in numerous fields such as engineering processes (wastewater treatment, gas-liquid reactors, etc.) or in the treatment of environmental problems (e.g. reaeration of lakes). As pointed out by Asher and Litchendorf (2008), PLIF has several advantages over other techniques (no flow disturbance, high optical resolution to visualize the phenomenon) and has already contributed to a better characterization of gas-liquid transport: Walker and Peirson (2008), Herlina and Jirka (2004) and Woodrow and Duke (2001) determined liquid side mass transfer coefficients across flat and wavy interfaces. Falkenroth (2007), Takehara and Etoh (2002) and Münsterer and Jähne (1998) focused on the thickness of the diffusion sublayer of different gases in water. Recently, Francois et al. (2011) have worked on the quantification of mass transfer around a single oxygen bubble rising in a quiescent liquid. However, to the best of our knowledge, there are no experiments related to the determination of molecular diffusivities of gases in liquid by PLIF. Some authors have pointed out the difficulties associated with such experiments: small thickness of the diffusion sublayer, which makes high-resolution optical systems necessary (Falkenroth 2007), optical blurring at the interface, issues due to natural or forced convection, etc. The purpose of this paper is to present a new method for visualizing and quantifying oxygen diffusion across a flat air-liquid interface by PLIF with inhibition (PLIFI). Particle Image Velocimetry (PIV) experiments performed to determine hydrodynamic profile in the liquid phase are also reported.

* Corresponding author melanie.jimenez@insa-toulouse.fr 


\section{Materials and methods}

\subsection{Materials}

\subsubsection{Hele-Shaw cell}

The experimental set-up is described in Figure 1. It was composed of a transparent Hele-Shaw cell and an optical system. The Hele-Shaw cell [1] was $5 \mathrm{~cm}$ high, $5 \mathrm{~cm}$ wide and $0.2 \mathrm{~cm}$ thick. The cell sides were made of PMMA (Polymethyl Methacrylate) with two gas orifices placed $1 \mathrm{~cm}$ below the top of the cell to allow the gas to flow [2].

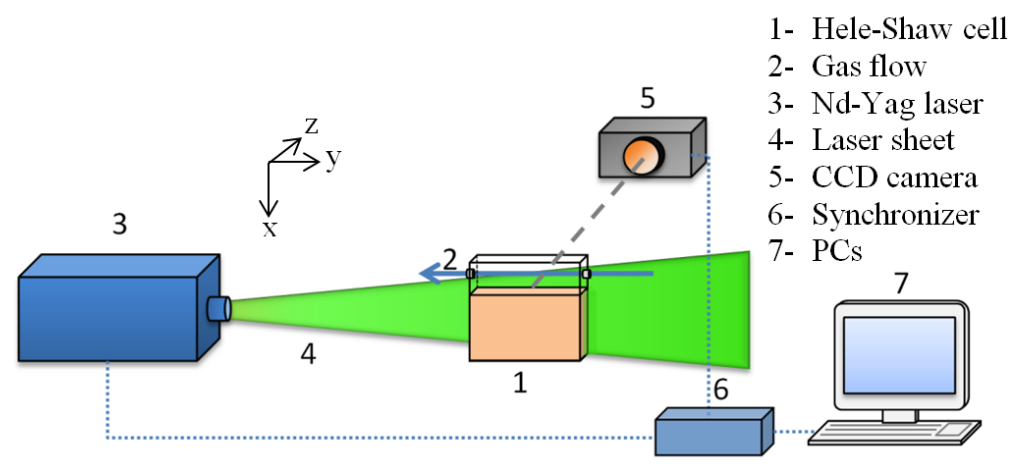

Figure 1. Experimental set-up

\subsubsection{Liquid medium}

The cell was filled to a height of approximately $3 \mathrm{~cm}$ with the liquid to be studied. An air flow of about $0.01 \mathrm{~L} / \mathrm{s}$ was passed tangentially over the liquid surface without disrupting it. As in the work of Francois et al. (2011), the liquid medium consisted of $80 \%$ by mass of deionized water, $25 \mathrm{mg} / \mathrm{L}$ of ruthenium complex, $\mathrm{C}_{72} \mathrm{H}_{48} \mathrm{~N}_{8} \mathrm{O}_{6} \mathrm{Ru}$, to observe the fluorescence, and $20 \%$ by mass of ethanol to solubilize the fluorescent dye. The viscosity and density of the liquid medium were respectively $\mu=0.0010$ Pa.s and $\rho=$ $970 \mathrm{~kg} \cdot \mathrm{m}^{-3}$ and were measured with a Haake VT550 Viscotester (Thermo scientific, Thermo Fisher Scientific Inc) and with a pycnometer (Brand Duran, V=25 cm3).

\subsubsection{PLIF}

PLIF is based on the excitation by a laser sheet of a fluorescent dye (ruthenium complex) that emits photon when it returns to its fundamental state. In presence of a quencher, such as oxygen, the fluorescence of the dye is inhibited due to collision with the quencher. These experiments focused on the inhibition of the ruthenium complex fluorescence by the oxygen diffusing in the liquid medium. The laser sheet was produced by a Nd:Yag laser [3] (Quantel, $\lambda=532 \mathrm{~nm}, 10 \mathrm{~Hz}, 200 \times 2 \mathrm{~mJ}$ ). The laser sheet crossed the Hele-Shaw cell vertically, along the xy plane. A Charge-Coupled-Device (CCD) camera [5] (Imager Intense, LaVision, Germany, 12 bits, $1040 \times 1376$ pixels $^{2}$ ) was placed perpendicularly to the $\mathrm{Nd}$ :Yag laser, in front of the xy plane, in order to record the fluorescence level at the gas-liquid interface. A 105-mm objective (Micro-Nikkor $105 \mathrm{~mm} \mathrm{f/8,} \mathrm{Nikon)} \mathrm{and} \mathrm{a} 570 \mathrm{~nm}$ high-pass filter, which avoided the laser light being recorded, were added to the digital camera. The Nd:Yag laser and the digital camera were synchronized by a Programmable Trigger Unit (LaVision) [6]. Data were then collected using specific acquisition software (Davis 7.2, LaVision).

\subsubsection{PIV}

Particle Image Velocimetry was used to observe hydrodynamic flow fields of the liquid phase in the same conditions as those of the experiments. The PIV experimental set-up was similar to that for the PLIF technique except for the Nd:Yag laser used (Dantec Dynamics Big Sky-Twins, $\lambda=532 \mathrm{~nm}, 15 \mathrm{~Hz}, 30 \times$ $2 \mathrm{~mJ}$ ). The software used to analyze the results was Flow Manager Version 4.60. The tracer particles were silver-coated hollow glass spheres made by Dantec Dynamics. 


\subsection{Experimental procedure}

The first step was the determination of the calibration curve for the conversion of fluorescence level (recorded as grey value in Davis) to oxygen concentration using the Stern-Volmer equation (1) (Stern and Volmer 1919).

$$
\frac{I_{Q}}{I_{0}}=\frac{1}{1+K_{S V}[Q]}
$$

where $\mathrm{K}_{\mathrm{sv}}$ is the Stern-Volmer constant (L/mg), [Q] the quencher concentration $(\mathrm{mg} / \mathrm{L})$, $\mathrm{I}_{\mathrm{Q}}$ and $\mathrm{I}_{\mathrm{o}}$ the fluorescence intensities in presence and absence of quencher respectively. This curve was determined by registering the fluorescence level of a solution with a known oxygen concentration. In this work, experimental points fitted the Stern-Volmer equation with a square correlation coefficient of $99.5 \%$.

In the second step, for each experiment, the liquid medium was completely deoxygenated with nitrogen. A nitrogen flow was generated above the interface in the Hele-Shaw cell so that the solution was not oxygenated. After a few minutes, the gas flow was switched from nitrogen to air at a flow rate of 0.01 L/s. A laser sheet was then produced approximately every 30 seconds. For each laser beam, five images were recorded simultaneously. The duration of experiment was limited to about 30 minutes to avoid evaporation issues. Experiments were carried out at $20^{\circ} \mathrm{C}$ under atmospheric pressure.

\subsection{Method for determining oxygen diffusivity}

The experiments carried out in the Hele-Shaw cell could be characterized by a two-dimensional (xy) problem, with an oxygen concentration gradient only in the x-direction and with no convection in this direction.

The second form of Fick's law could thus be written as (2) (Crank 1975).

$$
\frac{\partial\left[O_{2}\right]}{\partial t}=D \frac{\partial^{2}\left[O_{2}\right]}{\partial x^{2}}
$$

where $\left[\mathrm{O}_{2}\right]$ is the oxygen concentration $(\mathrm{mg} / \mathrm{L})$ and $\mathrm{D}$ the oxygen diffusivity in the liquid medium $\left(\mathrm{m}^{2} / \mathrm{s}\right)$. Considering a semi-infinite solution, the analytical solution of (2) is given by (3)

$$
\frac{\left[O_{2}\right]-\left[O_{2}\right]_{0}}{\left[O_{2}\right]_{s}-\left[O_{2}\right]_{0}}=1-\operatorname{erf}\left(\frac{x}{2 \sqrt{D t}}\right)
$$

where $\left[\mathrm{O}_{2}\right]_{\mathrm{s}}$ is the saturation concentration at the gas-liquid interface $(\mathrm{mg} / \mathrm{L}),\left[\mathrm{O}_{2}\right]_{\mathrm{o}}$ the concentration far away from the interface $(\mathrm{mg} / \mathrm{L})$.

By estimating the oxygen concentration as a function of the distance from the interface, $x$, and of the time, $t$, and assuming (3) to be valid, the oxygen diffusivity could be determined by parametric identification.

\subsection{Image processing method}

Before extracting the concentration profile of oxygen in the liquid medium, PLIF image processing was required. The main steps of this processing consisted of averaging the five images recorded at each laser beam, detecting the interface in the resulting image, removing the laser beam absorption using the BeerLambert theory and denoising images with a filter covering 5 pixels in the x-direction and 20 in the ydirection. More details of the treatment processes are presented in Herlina and Jirka (2004). The averaged oxygen concentration at each x-position was then determined by averaging concentration values along the $\mathrm{x}$-direction. 


\section{Data analysis and results}

\subsection{Oxygen diffusion visualization}

An example of PLIF results is shown in Figure 2. The evolution of the diffusion is represented at three different times. Due to the slowness of the phenomenon, it is preferable to wait about twenty minutes $t_{0}$ in order to visualize a measurable evolution. The darker the area, the stronger the fluorescence inhibition and thus the higher the oxygen concentration. The diffusion of oxygen in the liquid medium is clearly visible. This diffusion was faster at the beginning of the experiment and became very slow after a few minutes. This is due to the variation of the oxygen concentration gradient which is stronger at the beginning when the solution is fully deoxygenated to become slower once oxygen has transferred in the solution.
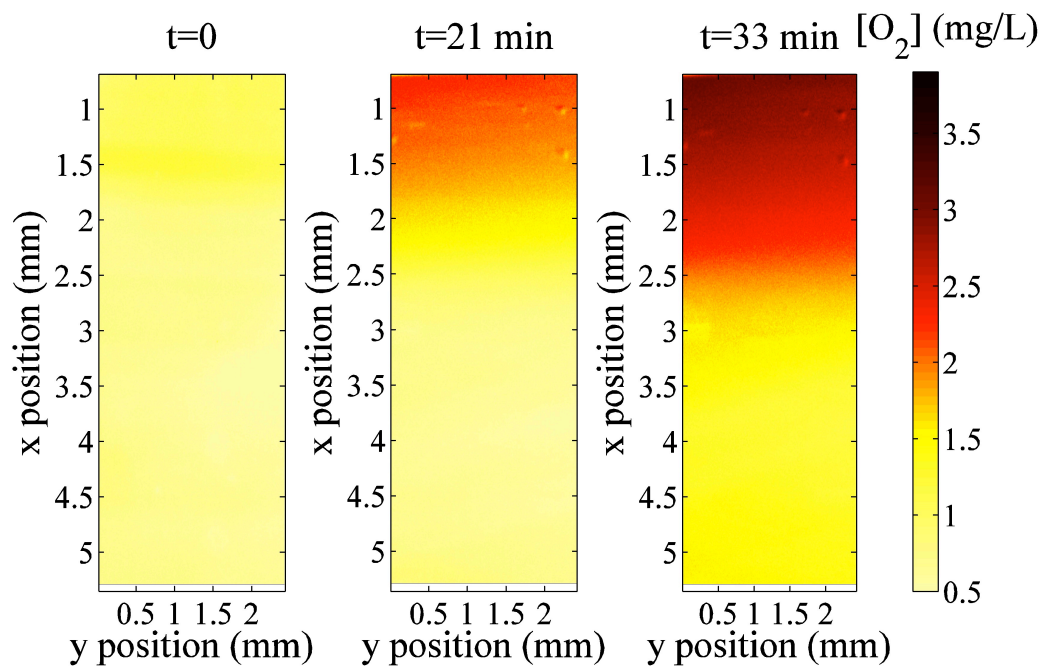

Figure 2. Visualization of oxygen diffusion in the liquid. The color bar represents oxygen concentration $(m g / L)$

Because of reflection at the gas-liquid interface, the oxygen concentration profile close to the interface was not visible and information was not available for about $0.3 \mathrm{~mm}$ in the $\mathrm{x}$-direction. The special cares presented by Walker and Peirson (2008) in order to improve the interface localisation are not applicable in our case due to the impossibility to achieve the geometric calibration of an inclined camera in a HeleShaw cell. This explains the maximum concentration value $\mathrm{c}_{\max }$ in Figure 2, about $5 \mathrm{mg} / \mathrm{L}$ instead of the expected saturation concentration at $20^{\circ} \mathrm{C},\left[\mathrm{O}_{2}\right]_{\mathrm{s}}$, measured at $9.01 \mathrm{mg} / \mathrm{L}$ by probe in these experiments (Jamnongwong et al., 2010).

\subsection{Hydrodynamic flow fields in the liquid phase}

To check the assumption of no convection along the diffusion direction, PIV experiments were conducted in the same experimental conditions as the PLIF ones. Figure 3 represents the hydrodynamic flow field in the liquid phase. The global velocity field in the Hele-Shaw cell was a loop generated by the gas flowing over the interface (Fig 3a.). At first sight, it seemed that the liquid and the gas phases flowed in opposite directions.

However, numerical simulations revealed an air recirculation above the interface due to the geometry of the gas outlet. These simulations were performed with the software COMSOL multiphysics $3.5 \mathrm{a}$ using an incompressible Navier-Stokes model. 


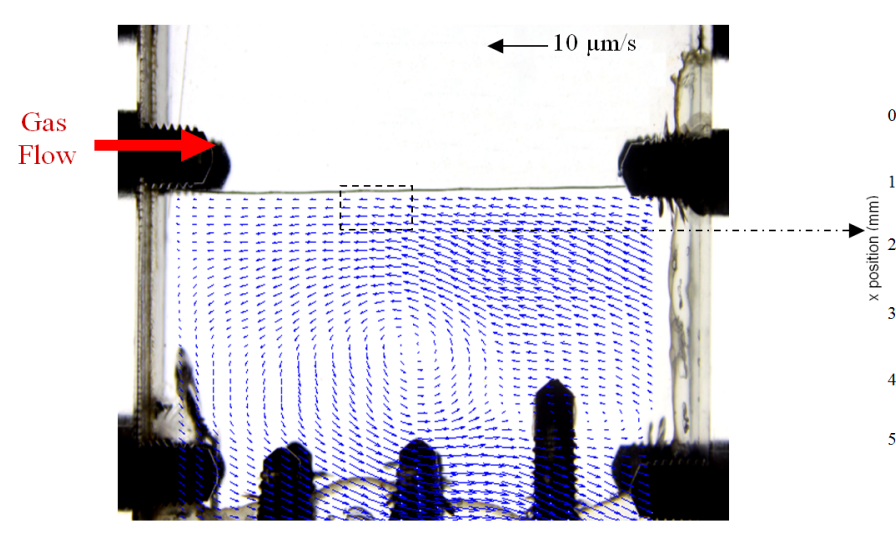

a

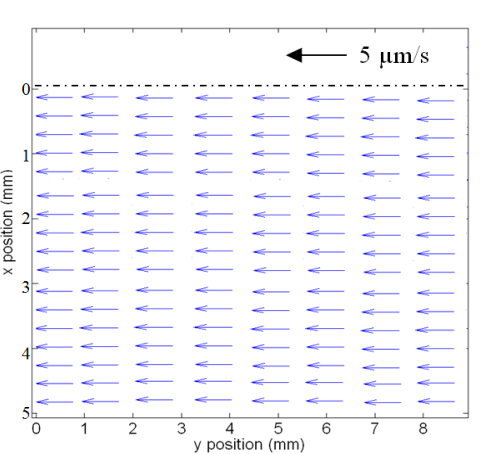

b

Figure 3. Velocity profile in the Hele-Shaw cell

Figure3. (b) represents the hydrodynamic flow field in the area where oxygen diffusion was observed. It was shown that the convection contribution along the $\mathrm{x}$-direction could be neglected and that the velocity field along the $y$-direction was quite weak $(<5 \mu \mathrm{m} / \mathrm{s})$. There was thus no perturbation along the diffusion direction. It is essential to note that the hydrodynamic field could differ strongly from the one depicted in Figure 3 depending on the air flow chosen and the liquid level. A correct selection of these parameters was of prime importance to avoid instabilities.

\subsection{Oxygen diffusivity determination}

Based on the oxygen concentration profile determined by PLIF, oxygen diffusivity could be estimated by fitting the experimental oxygen concentrations and the analytical expression (3) with a least-squares method. Theoretical and experimental points are compared in Figure 4.

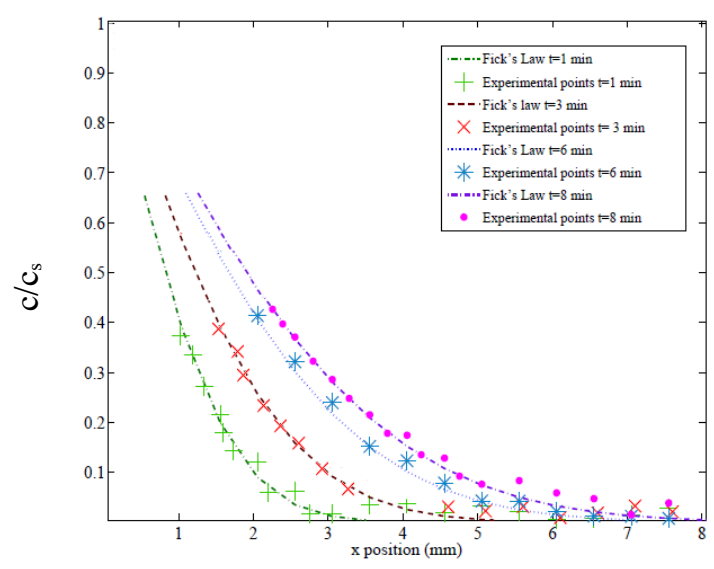

Figure 4. Oxygen concentration profile near the gas/liquid interface at different times $\left(t=1+t_{0}, 3+t_{0}, 6+t_{0}\right.$ and $8+t_{0}$ with $t_{0}=21 \mathrm{~min}$ )

Experimental data fitted the analytical expression (3) correctly even though convergence issues appeared for certain data. This new approach allowed the oxygen diffusivity to be determined as $1.94 \times 10^{-9} \mathrm{~m}^{2} / \mathrm{s}$. Table 1. illustrates the comparison between this estimated value and the common empirical correlations.

Table 1. Comparison of oxygen diffusivity in the liquid studied 


\begin{tabular}{lccl}
\hline & Experimental & Wilke et Chang $^{\mathrm{a}}$ & Scheibel $^{\mathrm{c}}$ \\
\cline { 2 - 4 } Oxygen diffusivity $\left(\mathrm{m}^{2} / \mathrm{s}\right)$ & $1.94 \times 10^{-9}$ & $1.994 \times 10^{-\varsigma}$ & $2.3 \times 10^{-9}$ \\
Error & $10 \%$ & $20 \% 0^{\mathrm{b}}$ & $<20 \% \mathrm{~d}^{\mathrm{d}}$ \\
\hline
\end{tabular}

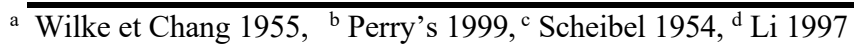

\section{Conclusion}

In this paper, a new and promising method for determining oxygen diffusivity across a flat interface has been presented. PLIF images provided a new qualitative insight into the oxygen diffusion in the liquid and enabled the oxygen concentration profile to be determined near an air-liquid interface. For the first time in literature, an experimental setup has been proposed to visualize and quantify a mass transfer process purely diffusive. This method is then efficient and promising, as the proposed protocol for this new diffusion coefficient measurement. The analytical expression of the problem gave an oxygen diffusivity value in accordance with the literature. PIV results showed that, under specific conditions, the hydrodynamic profile in the Hele-Shaw cell did not disrupt the diffusion process and thus that the mathematical representation of the problem was valid. Yet some points of this method are questionable. First, the introduction of the fluorescent dye and ethanol into the liquid medium changed the initial liquid properties. It is usually assumed that the fluorescent dye has a negligible effect on the diffusion so further works will focus on the use of a dye that is directly soluble in the liquid to be studied. Then, continuing improvements are expected in the mathematical resolution linking experimental points and theory to avoid convergence issues.

\section{References}

Asher, W.E. and Litchendorf, T.M., 2008, Visualizing near-surface concentration fluctuations using laser-induced fluorescence, Experiments in Fluids 46, no. 2 (8, 2008): 243-253.

Chu, C. R. and Jirka, G. H., 1992, Turbulent gas flux measurements below the air-water interface of a grid-stirred tank, Int. J. Heat Mass Transfer, 35,1957-1968

Crank, J., 1975, The mathematics of diffusion, Second Edition, Clarendon Press, Oxford.

Falkenroth, A., Degreif, K., Jähne, B., 2007, Visualisation of Oxygen Concentration Fields in the Mass Boundary Layer by Fluorescence Quenching, Transport at the Air Sea Interface pp. 59-72, 2077, Springer-Verlag, Heidelberg

Francois, J., Dietrich, N., Guiraud, P., Cockx, A., 2011, Direct measurement of mass transfer around a single bubble by micro-PLIFI, Chemical Engineering Science, doi:10.1016/j.ces.2011.01.049

Herlina and Jirka, G.H., 2004, Application of LIF to investigate gas transfer near the air-water interface in a gridstirred tank, Experiments in Fluids, Vol. 37, pp. 341-34

Jamnongwong, M., Loubiere, K., Dietrich, N., Hébrard, G., 2010, Experimental study of oxygen diffusion coefficients in clean water containing salt, glucose or surfactant: Consequences on the liquid-side mass transfer coefficients, Chemical Engineering Journal 165 (2010) 758-768

Li, J., and. Carr, P.W, 1997, Accuracy of empirical correlations for estimating diffusion coefficients in aqueous organic mixtures, Anal. Chem. 69, pp. 2530-2536

Münsterer, T. et Jähne, B., 1998, LIF measurements of concentration profiles in the aqueous mass boundary layer, Experiments in Fluids 25, no. 3 (8, 1998): 190-196

Perry's Chemical Engineers' Handbook-Seventh Edition, 1999, McGraw-Hill

Scheibel, E.G., 1954, Correspondence: liquid diffusivities. Viscosity of gases. Ind. Eng. Chem. 46 p. 2007

Stern, O. and Volmer, M., 1919, Über die Abklingzeit der Fluoreszenz, Physik. Zeitschr. 20 183-188

Takehara, K., Etoh, GT., 2002, A direct visualization method for $\mathrm{CO} 2$ gas transfer at water surface driven by wind waves, Geophys Monogr 127, Gas Transfer at Water Surfaces, pp 89-95

Walker J. et Peirson W., 2008, Measurement of gas transfer across wind-forced wavy air-water interfaces using laser-induced fluorescence, Experiments in Fluids 44, no. 2 (2, 2008): 249-259.

Wilke, C.R. and Chang, P.C., 1955, Correlation of diffusion coefficients in dilute solutions. Am. Inst. Chem. Eng. J. 1, p. 264

Woodrow, P.T. and Duke, S.R., 2001, Laser-Induced Fluorescence Studies of Oxygen Transfer Across Unsheared Flat and Wavy Air-Water Interfaces, Industrial \& Engineering Chemistry Research 40, no. 8 (4, 2001): 1985-1995. 